\title{
Microsurgical clipping of a giant vertebrobasilar junction aneurysm under hypothermic circulatory arrest
}

\author{
Ulas Cıkla, M.D., Kutluay Uluç, M.D., Mustafa K. Baskaya, M.D. \\ Department of Neurological Surgery, University of Wisconsin, Madison, Wisconsin
}

Giant posterior circulation aneurysms pose a significant challenge to neurovascular surgeons. Among various treatment methods that have been applied individually or in combination, clipping under hypothermic circulatory arrest $(\mathrm{HCA})$ is rarely used. We present a 62-year-old man who initially underwent coil occlusion of the right vertebral artery (VA) for a $2.5 \mathrm{~cm}$ giant vertebrobasilar junction (VBJ) aneurysm. His neurological condition had declined gradually and the aneurysm grew to $4 \mathrm{~cm}$ in size. The patient underwent clip reconstruction of giant VBJ aneurysm under HCA. His postoperative course was prolonged due to his preexisting neurological deficits. His preoperative Modified Rankin Score was 5 , and improved postoperatively to 3 at three and six months, and to 2 at one year.

The video can be found here: http://youtu.be/L53SiLV8eJY.

KEYWORDS hypothermic circulatory arrest; intracranial aneurysm; giant aneurysm; vertebral artery; microsurgical clipping; basilar artery; video 